\title{
Ambient aging effects on the effective energy gap of ZnO thin films
}

Cite as: J. Appl. Phys. 127, 245704 (2020); https://doi.org/10.1063/5.0010329

Submitted: 09 April 2020 . Accepted: 11 June 2020 . Published Online: 23 June 2020

C. Bridoux (D), G. D. Ruano, J. M. Ferreyra, and M. Villafuerte

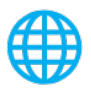

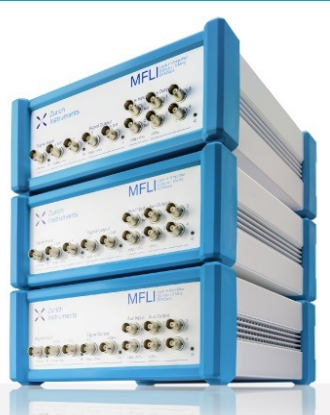

127, 245704 


\title{
Ambient aging effects on the effective energy gap of ZnO thin films
}

Cite as: J. Appl. Phys. 127, 245704 (2020); doi: 10.1063/5.0010329

Submitted: 9 April 2020 . Accepted: 11 June 2020 .

Published Online: 23 June 2020

G. Bridoux, $^{1, a)}$ (D) G. D. Ruano, ${ }^{2}$ J. M. Ferreyra, ${ }^{7}$ and M. Villafuerte $^{7}$

\author{
AFFILIATIONS \\ 'Laboratorio de Física del Sólido, INFINOA (CONICET-UNT), Facultad de Ciencias Exactas y Tecnología, \\ Universidad Nacional de Tucumán, 4000 San Miguel de Tucumán, Argentina \\ ${ }^{2}$ Instituto de Física del Litoral (IFIS), S3000GLN Santa Fe, Argentina
}

a) Author to whom correspondence should be addressed: gbridoux@herrera.unt.edu.ar

\begin{abstract}
Using photoconductance spectroscopy, we have studied the influence of different types of thermal annealing on epitaxial $\mathrm{ZnO}$ thin films where band bending effects play a major role. Once the film is exposed to ambient air conditions after a simple thermal annealing in oxygen at $600^{\circ} \mathrm{C}$, the effective energy gap is stable with a value of $\simeq 3.15 \mathrm{eV}$, while after a corresponding annealing in vacuum and subsequent air exposure, it starts at $\simeq 3.24 \mathrm{eV}$, and then it evolves along the days until it reaches the bulk energy gap value of $\mathrm{ZnO}$. By means of valence band $\mathrm{x}$-ray photoemission spectroscopy (XPS), we have confirmed that these phenomena are related via the Franz-Keldysh effect to a downward band bending in the former case and a time dependent upward band bending in the latter one that slowly tends to a flat band condition, tracking the behavior observed in the effective energy gap. Core level XPS measurements suggest that for each type of thermal annealing, a different adsorption kinetics of water and hydrogen take place.
\end{abstract}

Published under license by AIP Publishing. https://doi.org/10.1063/5.0010329

\section{INTRODUCTION}

$\mathrm{ZnO}$ is an earth abundant wide bandgap semiconductor with a large exciton binding energy, which makes it a suitable candidate for the next generation of transparent optoelectronics devices. ${ }^{1-3}$ In particular, its polar surfaces perpendicular to the c-axis $[(000 \overline{1})$ $\mathrm{O}$-polar and (0001) Zn-polar] try to reconstruct, dissociate water, and adsorb the resulting hydrogen or accumulate free carriers from the bulk in order to become stable, ${ }^{4-7}$ which are very appealing properties for catalysis or gas sensing. ${ }^{8-10}$ Since these effects influence the amount of accumulated charge on the surface, they can dramatically affect the band bending over a distance of tens of nanometers. ${ }^{11,12}$ This has triggered intensive research during the last years with the aim of tuning the band bending properties of $\mathrm{ZnO}$ single crystals. ${ }^{5,13,14}$ On the other hand, the improvement of thin films fabrication techniques in the last two decades ${ }^{15}$ offers an opportunity to study the band bending in nano-structures where these effects are expected to be enhanced ${ }^{16}$ and have an impact not only on surface photoemission techniques ${ }^{5,13,14,17}$ but also on other properties like optical absorption or photoconductivity. ${ }^{18}$

In this work, we confirm that photoconductivity spectra are dramatically affected in the case of high quality $\mathrm{ZnO}$ thin films.
From these results, the effective energy gap, $E_{G}$, can be extracted, which is an opto-electronic gap defined by the band to band transition of the conducting photoelectrons, see the sketch of Fig. 1(a). $E_{G}$ was tuned by means of simple thermal annealing in oxygen or vacuum atmospheres. After the annealing treatments, the film is exposed to ambient air conditions, $E_{G}$ can rapidly reach a stable value or can evolve along several days (until a saturation value is attained) depending on the type of thermal annealing. Furthermore, the photoconductivity measurements were complemented by valence band $\mathrm{x}$-ray photoelectron spectroscopy (valence band XPS). The results confirm that changes in the band bending produced by a combination of different adsorption kinetics and bulk carrier concentrations are responsible for the observed aging effects.

\section{EXPERIMENTAL}

$\mathrm{ZnO}$ films were grown by pulsed laser deposition (PLD) on (0001) sapphire substrates (dimensions: $5 \times 5 \times 0.5 \mathrm{~mm}^{3}$ ) at $550{ }^{\circ} \mathrm{C}$ with an oxygen pressure of 0.05 mTorr, see details elsewhere. ${ }^{18}$ The resulting films grew epitaxially in the [0001] direction ${ }^{18}$ with thickness values around $t \simeq 54 \mathrm{~nm}$. The results were similar for all the 
films studied. Two types of post-growth annealing at $600^{\circ} \mathrm{C}$ during $1 \mathrm{~h}$ were performed in these films: one of them using a base pressure of $\sim 1 \times 10^{-6}$ Torr (labeled as TA-Vac) and the other one with an oxygen pressure of 100 Torr (labeled as TA- $\mathrm{O}_{2}$ ). After each thermal annealing, the films were exposed to air under ambient conditions and monitored day by day using photoconductance spectroscopy measurements at room temperature as described elsewhere. ${ }^{18}$ This tracing was complemented by core level and valence band $\mathrm{x}$-ray photoelectron spectroscopy measurements ${ }^{5}$ using an incident energy of $h v=1486.6 \mathrm{eV}$. In the latter study, after air exposure of the sample, the film had to be placed in a UHV chamber (base pressure $\sim 10^{-10}$ Torr) in order to take a XPS spectrum. Then, it was removed from the chamber and again exposed to air before taking a new spectrum. Photoelectrons were detected using a Specs Phoibos 150 hemispherical electron energy analyzer with the detector axis located in a position normal to the film surface. All our facilities are located in a room with controlled temperature and humidity. The relative humidity ranges between $35 \%$ and $40 \%$ and the temperature between 21 and $23^{\circ} \mathrm{C}$.

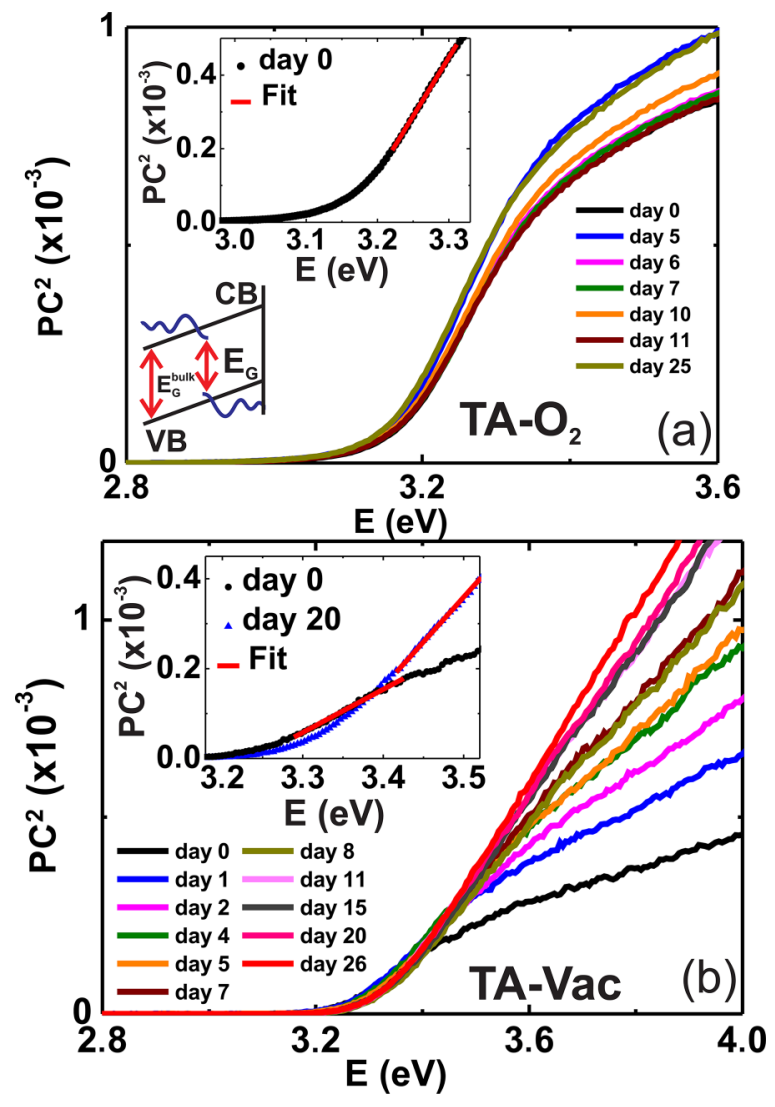

FIG. 1. $P C^{2}$ spectra monitored over days after air exposure for $\mathrm{TA}^{-\mathrm{O}_{2}}$ (a) and TA-Vac (b). The corresponding inset shows the linear fittings (red lines) of these curves after the first day of air exposure (day 0 ) and also after 20 days of air exposure in the case of TA-Vac [see the inset of panel (b)]. The sketch in panel (a) illustrates the Franz-Keldysh effect and the effective energy gap, $E_{G}$.

\section{RESULTS AND DISCUSSION}

Figure 1 shows the effects of the thermal annealing in oxygen (panel a) and vacuum (panel b) on photoconductivity spectra, defined as $P C=\left(\sigma-\sigma_{\text {dark }}\right) / \sigma_{\text {dark }}$, after exposing the sample to air. At first sight, it can be observed that the energy onset of the TA-O spectra is shifted to lower energies compared to the one in TA-Vac. Besides, while the $\mathrm{TA}-\mathrm{O}_{2}$ spectra barely change throughout the days, the TA-Vac's ones evolve with time. Since in a first approximation, $\mathrm{PC}$ is proportional to the optical absorption coefficient $^{18}$ and considering that $\mathrm{ZnO}$ has a direct energy gap $\left(E_{G}^{\text {bulk }} \simeq 3.3 \mathrm{eV}\right),{ }^{19}$ the linear relation $P C^{2} \propto E-E_{G}$ should be satisfied in a certain range of energies above the energy onset, ${ }^{20}$ see the inset of Fig. 1. From these fittings, the effective energy gap $E_{G}$ of the film can be extracted and monitored after each type of thermal annealing and their subsequent exposition to air [see Fig. 2(a)]. In this figure, an alternated sequence of the two types of thermal annealing on the same film is presented. As it can be observed, after a thermal annealing in oxygen (whose first measurement after air exposure is labeled as O) $E_{G}$ remains at a value of $\simeq 3.15 \mathrm{eV}$ (this occurs for both $\mathrm{O} 1$ and $\mathrm{O} 2$ within the

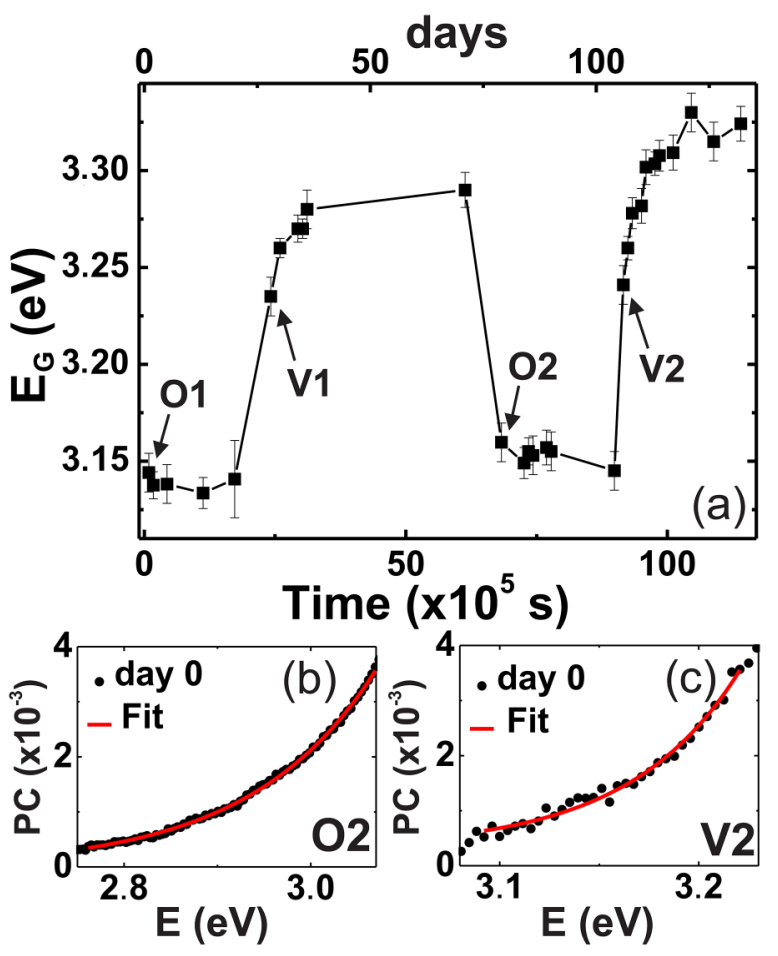

FIG. 2. (a) $E_{G}$ values as a function of time after air exposure during an alternate sequence of thermal annealing performed in the same sample. Labels 01 and $\mathrm{O} 2$ indicate the first measurement performed after the first and second thermal annealing in oxygen, respectively. Labels V1 and V2 indicate the first measurement performed after the first and second thermal annealing in vacuum, respectively. The PC spectra after a day of air exposition (day 0 ) for $\mathrm{O} 2$ and V2 are presented in (b) and (c), respectively. Their corresponding fittings using the Dow and Redfield expression are also shown (red curves). 
experimental error). On the other hand, after a thermal annealing in vacuum (whose first measurement after air exposure is labeled as V), $E_{G}$ starts with a value of $\simeq 3.24 \mathrm{eV}$ (which is the same value for both V1 and V2 within the experimental error), and then it slowly increases during the days until it reaches a saturation value close to $E_{G}^{b u l k}$.

On the other hand, after a TA-O $\mathrm{O}_{2}$, the conductivity in dark of the film is $\sigma_{\text {dark }}^{O_{2}}=51.43 \Omega^{-1} \mathrm{~m}^{-1}$, while after TA-Vac, $\sigma_{\text {dark }}^{\text {Vac }}=156.57 \Omega^{-1} \mathrm{~m}^{-1}$. This is a plausible result considering that a TA-Vac generates donor oxygen vacancies in the $\mathrm{ZnO}$ film that increases its electrical conductivity. These results confirm previous studies that suggest that oxygen vacancies are responsible for the $n$-type conductivity in $\mathrm{ZnO}^{21,22}$ This has been an issue of long debate during the last years since many reports ${ }^{23,24}$ predict a neutral character of oxygen vacancies in this material.

The fact that these energy gap values are lower compared to the bulk one is related to a strong influence of band bending along the film thickness direction. ${ }^{11}$ The typical width of an upward band bending in $\mathrm{ZnO}$ is around $d \sim 70 \mathrm{~nm},{ }^{25,26}$ which is similar to our film thickness value. This band bending allows Airy wave functions of electrons and holes to penetrate in the forbidden energy region below the minimum of the conduction band $(\mathrm{CB})$ and above the maximum of the valence band (VB), leading in this way to a reduction of the effective energy gap value compared to the bulk one. Besides, it also produces a shift of the optical absorption onset toward lower energies, known as the Franz-Keldysh effect, ${ }^{27,28}$ see the sketch of Fig. 1(a). The higher the band bending, the higher the shift of the onset and the lower the effective energy gap. In this situation, the absorption spectra (and hence the PC spectra) can be fitted in an energy region very close to the onset $^{16,18}$ using a model proposed by Dow and Redfield that considers the presence of a uniform electric field and it includes the effects of excitonic transitions. ${ }^{29}$ In this model, $P C \sim e^{E / \Delta E}$, where $\Delta E$ takes into account the energy gap reduction, and it measures the strength of the electric field that generates the band bending. ${ }^{16,18}$ Typical fittings of the PC spectra using this expression are presented in Figs. 2(b) and 2(c). In the case of oxygen annealing, $\Delta E \simeq 0.15 \mathrm{eV}$, while a day after the thermal annealing in vacuum $\Delta E \simeq 0.06 \mathrm{eV}$, in agreement with the corresponding differences between $E_{G}$ [Fig. 2(a)] and $E_{G}^{\text {bulk }}$. These results suggest that the band bending effect is stronger in the case of the thermal annealing in oxygen, whereas for the thermal annealing in vacuum, the band bending slowly decreases throughout the days until a flatband condition is reached.

A similar tracing after each type of annealing was performed using valence band XPS measurements [see Fig. 3(a)]. This technique allows us to extract the band bending values as follows. In each spectrum, the energy difference between the maximum of the $\mathrm{VB}$ and the Fermi level $E_{F},\left(\zeta=E_{V}-E_{F}\right)$, was extracted from the intersection of two linear fits [see Fig. 3(a)]., ${ }^{5,14}$ Then, the band bending $V_{B B}$ can be obtained as $V_{B B}=E_{G}^{b u l k}-\zeta-\xi$, where $\xi=E_{C}-E_{F}=\left(k_{B} T / q\right) \ln \left(N_{c} / \bar{n}\right) \quad$ with $N_{c}$ being the CB effective density of states $\left(N_{c}=2.94 \times 10^{18} \mathrm{~cm}^{-3}\right.$ for $\left.\mathrm{ZnO}\right), \bar{n}$ the carrier density obtained from Hall effect measurements in the film, ${ }^{30,31}$ and $E_{C}$ the minimum of the CB. In the case of TA-Vac, $\bar{n}_{V a c}=2.57 \times 10^{17} \mathrm{~cm}^{-3}$, while for TA-O , $\bar{n}_{\mathrm{O}_{2}}=8.46 \times 10^{16} \mathrm{~cm}^{-3}$. Figure $3(\mathrm{~b})$ shows the extracted $V_{B B}$ values of the film over the days after each type of thermal annealing and the subsequent air exposure. As it can be observed, the absolute values of $V_{B B}$ for TA-O $\mathrm{O}_{2}$ are higher than the ones for TA-Vac, confirming the results obtained for the effective energy gap $E_{G}$ in Fig. 2(a). Besides, the $V_{B B}$ values for TA- $\mathrm{O}_{2}$ and TA-Vac have opposite sign corresponding to an accumulation layer in the former case and a depletion layer in the latter ${ }^{5,11,32}$ (see Fig. 4). More important, for TA-Vac, the upward band bending starts at a value of $V_{B B}=+0.17 \mathrm{eV}$ after air exposure, and then it slowly decreases along the days toward a condition of flatband in accordance with the increase of $E_{G}$ toward $E_{G}^{b u l k}$ [see Figs. 2(a) and 3(b)].

In the case of an upward band bending, free electrons are attracted to the surface and this negative charge is compensated by an equivalent amount of ionized donor centers in the bulk, which are positively charged (mainly oxygen vacancies in our $\mathrm{ZnO}$ film,
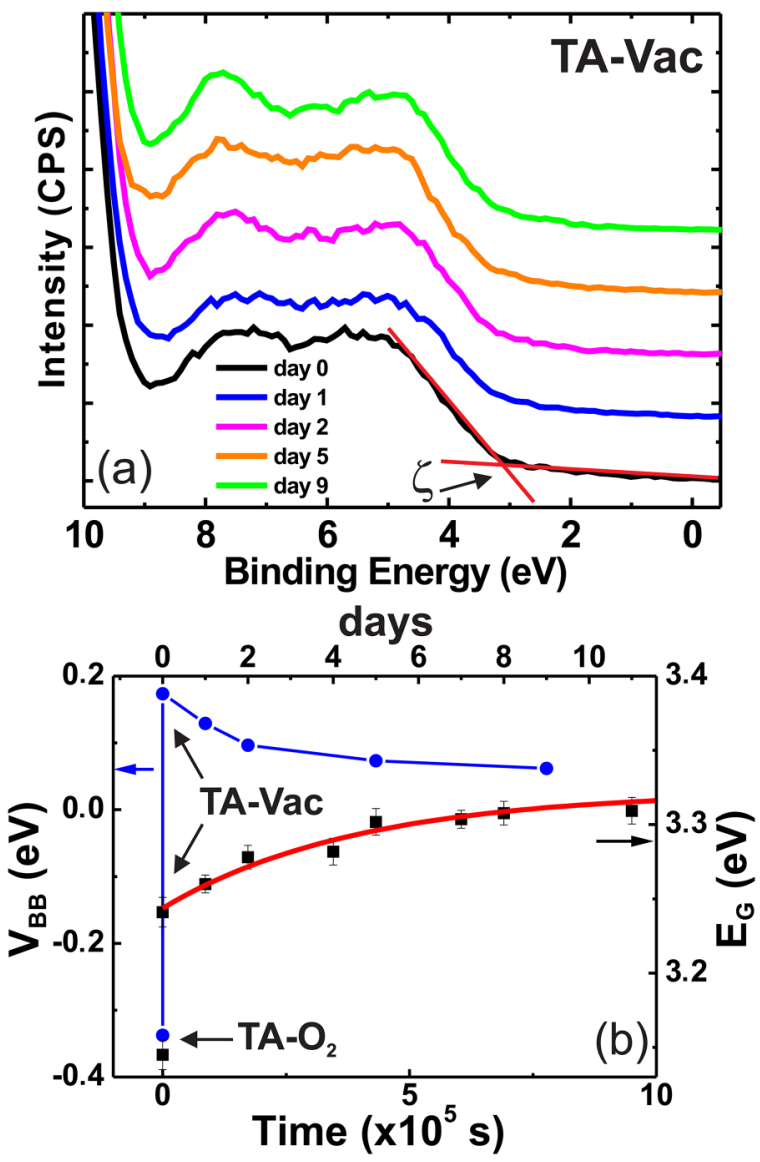

FIG. 3. (a) Valence band XPS spectra recorded throughout the days after air exposure in the case of TA-Vac. From the intersection of the two linear fits (red lines), $\zeta=E_{V}-E_{F}$ can be obtained. The spectra are shifted for clarity. (b) Extracted $V_{B B}$ values as a function of time for each type of thermal annealing (blue circles, left axis) and the time dependence of $E_{G}$ extracted from Fig. 2(a) (black squares, right axis). The aging effect observed after TA-Vac was quantified using an exponential fit (red curve). 

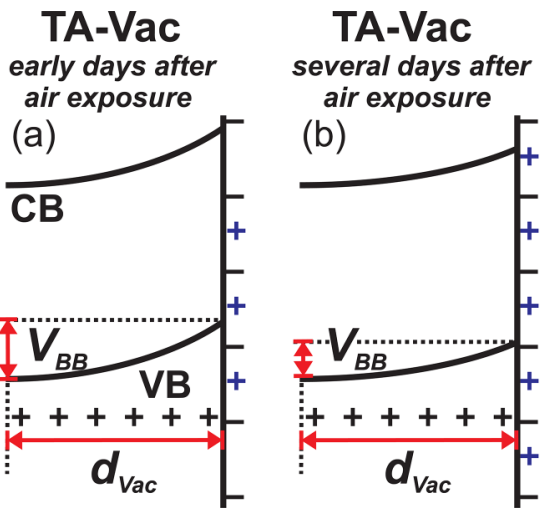

$\mathrm{TA}-\mathrm{O}_{2}$ air exposure

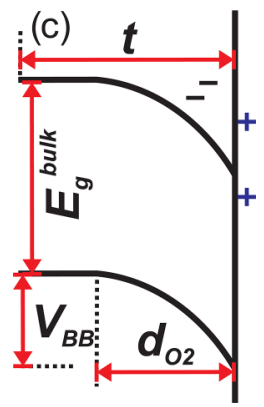

FIG. 4. Energy band diagram. (a) Early days of air exposure after TA-Vac. On the one hand, negative charge (free carriers) displace toward the surface trying to establish an upward band bending. This charge is compensated by the positive charge of ionized donor centers. On the other hand, since the film was exposed to air, the positive charge can be adsorbed on the film surface decreasing the upward band bending contribution of the free carriers. (b) After several days of air exposure, more positive charge was adsorbed on the film surface resulting in an even lower band bending. (c) When the film is exposed to air after $\mathrm{TA}-\mathrm{O}_{2}$, the positive charge on the surface due to adsorbed species is dominant (since the amount of negative free carriers after $\mathrm{TA}-\mathrm{O}_{2}$ is reduced), resulting in a downward band bending.

with density $\left.N_{d}\right)^{11,33,34}$ [see Figs. 4(a) and 4(b)]. Particularly, in O-polar terminated $\mathrm{ZnO}$ surfaces (like in most of the $\mathrm{ZnO}$ films fabricated by $\mathrm{PLD}^{35}$ ), free electrons move toward the surface in order to occupy dangling oxygen bonds on it. ${ }^{5,6,36}$ If the sample is exposed to air under ambient conditions, it is known that molecular water ${ }^{5,37}$ or hydrogen (emerging from the water dissociation on its surface ${ }^{5,14,38}$ ) can be adsorbed competing with the free electrons of the bulk for the occupation of dangling oxygen bonds. $^{4-6,14,32,38}$ As a consequence, positive charge (with a surface density $n^{\text {sup }}$ ) will start to be added to the surface decreasing the upward band bending contribution due to free carriers [modifications along the days are represented in Figs. 4(a) and 4(b)]. For TA-Vac, $n_{\text {Vac }}^{\text {sup }}$ can be estimated from the expression: ${ }^{11}$ $N_{d}-\left(n_{\text {Vac }}^{\text {sup }} / d_{\text {Vac }}\right)=2 \varepsilon_{r} \varepsilon_{0} V_{B B} / e^{2} d_{\text {Vac }}^{2}$, where the width of the depletion region is assumed to have the value of the film thickness, $d_{\text {Vac }}=t \simeq 54 \mathrm{~nm}, \varepsilon_{0}$ is the vacuum permittivity and $\varepsilon_{r}$ is the relative dielectric constant of $\mathrm{ZnO} .{ }^{19}$ Additionally, we approximate $N_{d} \simeq \bar{n}_{V a c}$. Just after air exposure, $V_{B B}=+0.17 \mathrm{eV}$ and, hence, $n_{\text {Vac }}^{\text {sup }} \simeq 1.1 \times 10^{12} \mathrm{~cm}^{-2}$, while at day $9 \quad\left(\simeq 7.7 \times 10^{5} \mathrm{~s}\right)$ $V_{B B}=+0.06 \mathrm{eV}$ and $n_{V a c}^{\text {sup }} \simeq 1.28 \times 10^{12} \mathrm{~cm}^{-2}$. This means a $\sim 15 \%$ enhancement of the surface protonization throughout the days.

On the other hand, after TA- $\mathrm{O}_{2}$, the concentration of oxygen vacancies is reduced and, therefore, the free electron densities too. In this situation, after air exposure the protonization of the surface will be a dominant contribution and a downward band bending will be established [see Fig. 4(c)]. This positive surface charge density, $n_{\mathrm{O}_{2}}^{\text {sup }}$, is compensated by an equivalent amount of negative charge accumulated in a region of width $d_{\mathrm{O}_{2}}$, being free electrons donated by the adsorbed species (e.g., chemisorbed hydrogens). ${ }^{32,39}$
In a first approximation, $n_{\mathrm{O}_{2}}^{\text {sup }}$ can be estimated as ${ }^{11}$ $n_{\mathrm{O}_{2}}^{\text {sup }} / d_{\mathrm{O}_{2}} \simeq 2 \varepsilon_{r} \varepsilon_{0} V_{B B} / e^{2} d_{\mathrm{O}_{2}}^{2}$, where we have taken an upper limit of $\sim 35 \mathrm{~nm}$ for $d_{\mathrm{O}_{2}}$ in accordance with the typical accumulation widths in $\mathrm{ZnO}^{32}$ Using $V_{B B}=-0.33 \mathrm{eV}$ from Fig. 3(b), it results in a value of $n_{\mathrm{O}_{2}}^{\text {sup }} \simeq 8.5 \times 10^{11} \mathrm{~cm}^{-2}$ and hence in a ratio $n_{\text {Vac }}^{\text {sup }} / n_{\mathrm{O}_{2}}^{\text {sup }} \simeq 1.3$, when it is compared with the initial protonization after TA-Vac.

In order to monitor the presence of adsorbed species in the film surface, O $1 s$ core level XPS spectra were recorded once the film was exposed to air after each type of thermal annealing. A typical spectrum is shown in Fig. 5(a). As it can be observed, the spectra were well fitted using three pseudo-Voigt functions after subtraction of a linear background. The full width at half maximum and the energetic separation of the three components were constrained during the fitting. The main component comes from bulk oxygen emission ( $\mathrm{O}$ atoms coordinated to their four nearest $\mathrm{Zn}$ atoms neighbors). The other two components may

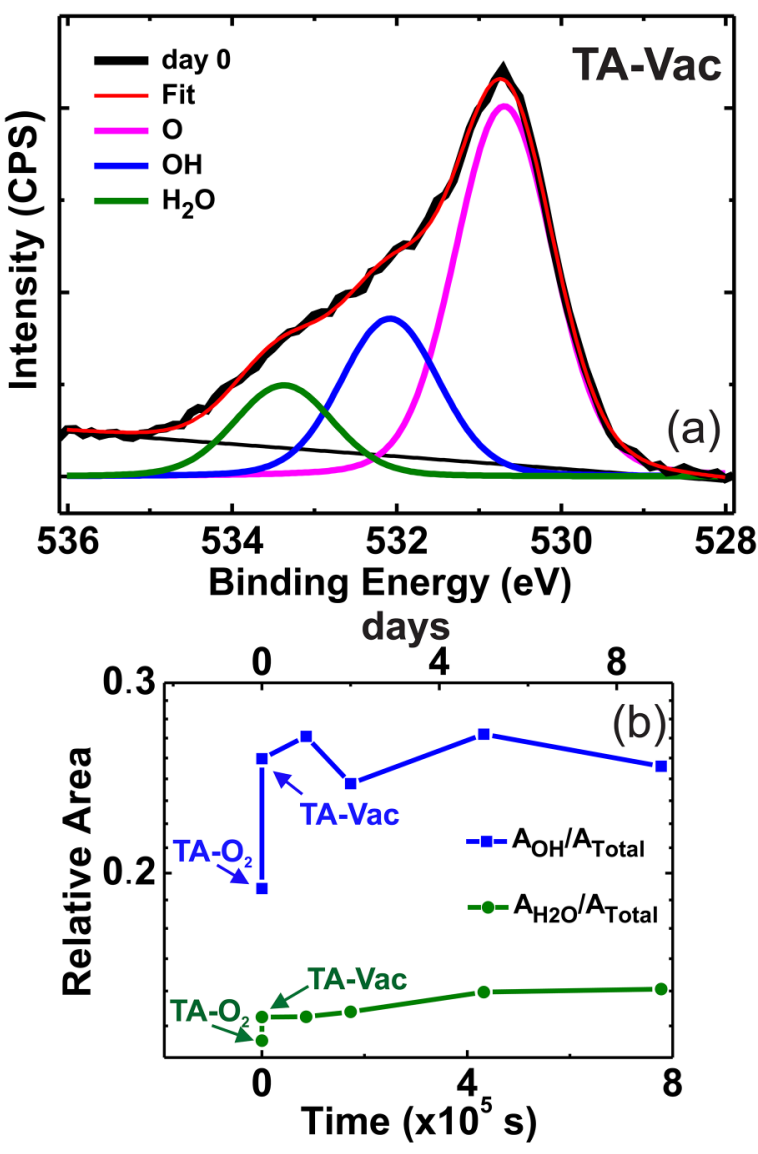

FIG. 5. (a) Typical $O 1 s$ core level XPS spectrum of the film after the annealing in vacuum and the subsequent air exposure during one day. The spectrum was well fitted using three components after linear background subtraction. (b) Relative peak area of each component throughout the days after $\mathrm{TA}-\mathrm{O}_{2}$ or TA-Vac was carried out on the film. 
come from surface atomic under-coordination. ${ }^{40}$ Previous core level XPS studies in $\mathrm{ZnO}^{4,5,13}$ propose that the second component (shifted to higher binding energy by $\sim 1.4 \mathrm{eV}$ ) is due to surface oxygen atoms coordinated to three $\mathrm{Zn}$ atoms and a surface terminating species. In the case of an O-polar surface, this species is known to be a hydrogen atom forming a hydroxyl $(\mathrm{OH})$ group with a surface oxygen of the film. ${ }^{4,-14}$ These XPS studies ${ }^{4,5,13}$ also suggest that the third component (around $\sim 533.5 \mathrm{eV}$ ) is associated with molecular water adsorbed either on the film surface or on top of these hydroxyl groups. ${ }^{37,41}$ In the present work we follow this assignment of the XPS peaks, where the corresponding peak areas of the $\mathrm{OH}$ and $\mathrm{H}_{2} \mathrm{O}$ components are labeled as $A_{\mathrm{OH}}$ and $A_{\mathrm{H}_{2} \mathrm{O}}$ respectively.

Figure 5(b) shows the relative peak areas of the $\mathrm{OH}$ and $\mathrm{H}_{2} \mathrm{O}$ components (denoted as $A_{\mathrm{OH}} / A_{\text {Total }}$ and $A_{\mathrm{H}_{2} \mathrm{O}} / A_{\text {Total }}$ respectively) along the days after TA- $\mathrm{O}_{2}$ and TA-Vac. As it can be observed, the initial surface protonization in TA-Vac (quantified by $\left.\left(A_{\mathrm{OH}}+A_{\mathrm{H}_{2} \mathrm{O}}\right) / A_{\text {Total }}\right)$ is higher than the one in $\mathrm{TA}-\mathrm{O}_{2}$ by a factor $\simeq 1.2$ in close agreement with the ratio $n_{\text {Vac }}^{\text {sup }} / n_{\mathrm{O}_{2}}^{\text {sup }} \simeq 1.3$ obtained from the previous band bending analysis. On the other hand, the increase of the surface protonization along the days (once the film was exposed to air after TA-Vac) is mainly due to an increase of molecular water, being this increment of around $\sim 6 \%$ at day 9 $\left(\simeq 7.7 \times 10^{5}\right.$ s) [see Fig. 5(b)].

Finally, the adsorption kinetics of these processes has been studied using the time dependent fraction of occupied surface sites, ${ }^{42} \theta(t) \simeq 1-e^{-t / \tau}$, where the relaxation time $\tau$ is connected with the activation energy for adsorption via $E_{a}=k_{B} T \ln (v \tau)$, with $^{43} v=S_{0} P / n_{\max }^{\text {sup }} \sqrt{2 \pi m k_{B} T}$. $S_{0}$ represents the trapping probability, $n_{\max }^{\text {sup }}$ the maximum surface density of adsorbed particles, $P$ is the partial pressure of this gas of particles and $m$ the mass of one of them. In the case of TA-Vac, $\tau$ can be extracted from an exponential fit to the data of Fig. 3(b) if a correlation between $E_{G}(t)$ and $\theta(t)$ is assumed, resulting in a value of $\tau \simeq 3.9 \times 10^{5} \mathrm{~s}$ (approximately 4.5 days). Besides, $n_{\max }^{\text {sup }}$ can be obtained from the band bending expression assuming that $V_{B B}=0$. It results in $n_{\text {max }}^{\text {sup }} \simeq N_{d} d_{V a c} \simeq \bar{n}_{V a c} d_{V a c} \simeq 1.3 \times 10^{12} \mathrm{~cm}^{-2}$. Taking $S_{0} \simeq 0.8$ for water in O-polar $\mathrm{ZnO}$ surface, ${ }^{38} P \simeq 24$ Torr as the partial water vapor pressure at normal conditions and $m \simeq 2.98 \times 10^{-26} \mathrm{~kg}$ as the mass of a water molecule, we obtain an activation energy of $E_{a} \simeq 0.9 \mathrm{eV}$. This high value of $E_{a}$ strongly suggests that a chemisorption process plays a relevant role on this aging effect ${ }^{43}$ (e.g., adsorption of hydrogen due to water dissociation ${ }^{5,14,38}$ ). Although this aging effect could not be observed in the case of $\mathrm{TA}^{-} \mathrm{O}_{2}$, we propose the following hypothesis: It is plausible that the aging effect is also occurring in TA- $\mathrm{O}_{2}$ with relaxation times of less than 1 day, which is the minimum time step employed in our experiments. This would involve activation energies that are a fraction of the value found for TA-Vac and, therefore, a surface with greater avidity for adsorbed species (or equivalently with deeper binding energy levels, $E_{B}$ ), see the sketch of Fig. 6(a). In $\mathrm{ZnO}$, it is known that adsorbed species like hydrogen generate shallow donor levels close to the surface. ${ }^{32,44}$ These electrons donated by these adsorbed hydrogens balance the excess of positive charge on the surface. These hydrogen levels can be represented by unperturbed molecular orbitals (the highest occupied one being labeled as $E_{\text {НОмO) }}$ and in terms of them the resulting bonding states $E_{B}$ can be
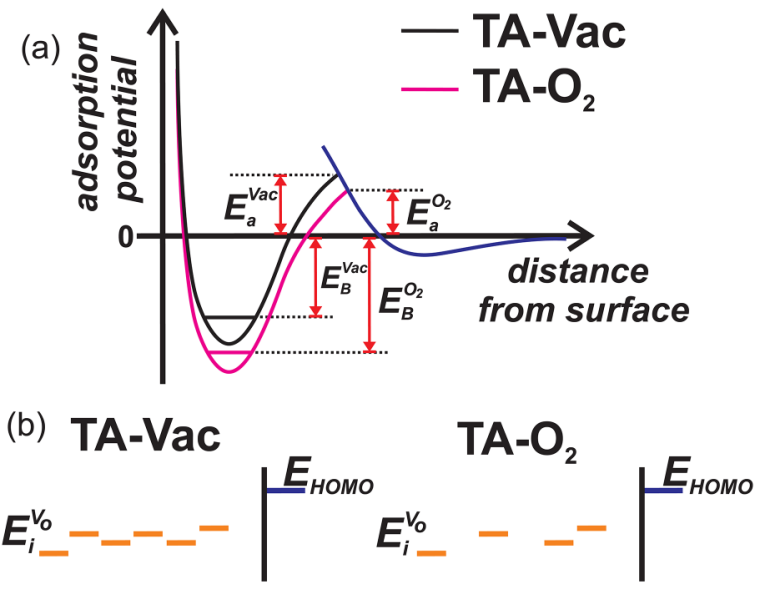

FIG. 6. (a) Qualitative shape of the adsorption potential as a function of the distance of the adsorbed species from the solid surface. After air exposure, the film annealed in oxygen presents a deeper bonding energy $E_{B}$ (and a lower activation energy $E_{a}$ ) than the one annealed in vacuum. The corresponding values of $E_{a}$ depend on the intersection of these curves with the physisorption potential (blue curve). ${ }^{43}$ (b) Energy levels in the case of TA-Vac (left panel) and $\mathrm{TA}^{-} \mathrm{O}_{2}$ (right panel). A higher density of levels with energies $E_{i}^{V_{0}}$ (due to oxygen vacancies) is generated in the case of TA-Vac, leading to an increased contribution to the second term of Eq. (1) compared with $\mathrm{TA}^{-\mathrm{O}_{2}}$

written as: ${ }^{45}$

$$
E_{B} \simeq E_{B}^{0}+\sum_{E_{i}^{V_{O}}>E_{F}} \frac{N_{i}\left|U_{H, i}^{V_{O}}\right|^{2}}{E_{H O M O}-E_{i}^{V_{O}}}
$$

where $E_{B}^{0}$ (with $E_{B}^{0}<0$ ) represents the main contribution to $E_{B}$ without including effects due to oxygen vacancies. We assume that this term is more or less the same for both types of treatments. $E_{i}^{V_{O}}$ is the energy of the $i^{\text {th }}$ level produced by oxygen vacancies in the film ${ }^{33,34}$ and $N_{i}$ is the total number of levels that share the same energy value $E_{i}^{V_{O}}$ [see Fig. 6(b)]. $\left|U_{H, i}^{V_{O}}\right|^{2}$ are interaction matrix elements between these levels and $E_{\text {HOMO }}$. While $E_{B}^{0}$ contributes negatively to $E_{B}$, the summation (where oxygen vacancies levels intervene) provides a positive contribution counterbalancing a bit the effect of $E_{B}^{0}$ and reducing in this way the depth of $E_{B}$ [see Fig. 6(b)]. Since during TA-Vac a higher density of levels (due to oxygen vacancies) is generated compared to TA- $\mathrm{O}_{2}$, the former annealing will provide an increased contribution to the summation in Eq. (1), reducing in this way the depth of $E_{B}$ compared with TA-O ${ }_{2}$, see Figs. 6(a) and 6(b).

\section{CONCLUSION}

$\mathrm{ZnO}$ thin films provide a platform to study enhanced band bending effects since the typical band bending width is similar to the film thickness value. In this way, parameters like the effective energy gap become very sensitive to modifications of the band bending properties due to the Franz-Keldysh effect. In the present work, we have studied how simple thermal annealing (in oxygen or 
vacuum) can affect the surface adsorption properties and the carrier concentration of a $\mathrm{ZnO}$ film, as detected by core level XPS and electrical transport measurements, respectively. As a consequence, band bending properties can be modified (as valence band XPS spectra show) resulting in an initial upward band bending that slowly tends to a flatband condition after a vacuum annealing and a stable downward band bending value in the case of oxygen annealing. These valence band XPS findings are in accordance with the behavior of the effective energy gap extracted using a different technique, that is, photoconductance spectroscopy. More important, after a vacuum annealing and the subsequent air exposure, $E_{G}$ evolves in time toward the $E_{G}^{b u l k}$ value for couple of days, tracking the behavior of the band bending toward a flatband condition. This combination of complementary techniques is very appealing not only because photoconductivity is a key optoelectronic property but also because we have been able to monitor aging effects on the band bending and hence in $E_{G}$ with the importance that it represents for researchers who want to design optoeletronic devices based on $\mathrm{ZnO}$ nano-structures. Finally, it is worth to mention that our route of tuning the effective energy gap (that we call optoelectronic gap) of $\mathrm{ZnO}$ thin films via the Franz-Keldysh effect differs from other studies where the bandgap is indeed modified by skin quantum entrapment based on the bond-order-length-strength (BOLS) correlation mechanism. ${ }^{46}$

\section{ACKNOWLEDGMENTS}

This work was supported by PIP No. 585, SCAIT No. E653CX, PICT No. 2016-3356, SNMAG, and SINALA facilities. We thank G. Zampieri and A. Butera.

\section{DATA AVAILABILITY}

The data that support the findings of this study are available from the corresponding author upon reasonable request.

\section{REFERENCES}

1'Ü. Özgür, D. Hofstetter, and H. Morkoç, Proc. IEEE 98, 1255 (2010).

${ }^{\mathbf{2}}$ H. Frenzel, A. Lajn, H. von Wenckstern, M. Lorentz, F. Schein, Z. Zhang, and M. Grundmann, Adv. Mater. 22, 5332 (2010).

${ }^{3}$ R. Schlesinger, F. Bianchi, S. Blumstengel, C. Christodoulou, R. Ovsyannikov, B. Kobin, K. Moudgil, S. Barlow, S. Hecht, S. R. Marder, F. Henneberger, and N. Koch, Nat. Commun. 6, 6754 (2015).

${ }^{4}$ J. V. Lauritsen, S. Porsgaard, M. K. Rasmussen, M. C. R. Jensen, R. Bechstein, K. Meinander, B. S. Clausen, S. Helveg, R. Wahl, G. Kresse, and F. Besenbacher, ACS Nano. 5, 5987 (2011).

${ }^{\mathbf{5}}$ R. Heinhold, G. T. Williams, S. P. Cooil, D. A. Evans, and M. W. Allen, Phys. Rev. B 88, 235315 (2013).

${ }^{6}$ S. Erker, P. Rinke, N. Moll, and O. T. Hofmann, New J. Phys. 19, 083012 (2017).

${ }^{7}$ M. Iachella, J. Cure, M. Djafari Rouhani, Y. Chabal, C. Rossi, and A. Estève, J. Phys. Chem. C 122, 21861 (2018).

${ }^{8}$ Y. H. Lu, S. P. Russo, and Y. P. Feng, Phys. Chem. Chem. Phys. 13, 15973 (2011). ${ }^{9}$ M. Reli, M. Edelmannová, M. Šihor, P. Praus, L. Svoboda, K. Kutláková Mamulová, H. Otoupalíková, L. Čapek, A. Hospodkov, L. Obalová, and K. Kočí, Int. J. Hydrogen Energy 40, 8530 (2015).

${ }^{10}$ S.-i Fujita, H. Mitani, C. Zhang, K. Li, F. Zhao, and M. Arai, Mol. Catal. 442, 12 (2017).

${ }^{11}$ Z. Zhang and J. T. Yates Jr., Chem. Rev. 112, 5520 (2012).
12J. M. Ferreyra, G. Bridoux, M. Villafuerte, B. Straube, J. Zamora, C. A. Figueroa, and S. P. Heluani, Sol. State Commun. 257, 42 (2017).

${ }^{13}$ R. Heinhold, S. P. Cooil, D. A. Evans, and M. W. Allen, J. Phys. Chem. C 118, 24575 (2014).

${ }^{14}$ A. R. McNeill, A. R. Hyndman, R. J. Reeves, A. J. Downard, and M. W. Allen, ACS Appl. Mater. Interfaces 8, 31392 (2016).

${ }^{15}$ J. Heber, Nature 459, 28 (2009).

${ }^{16}$ A. Cavallini, L. Polenta, M. Rossi, T. Stoica, R. Calarco, R. J. Meijers, T. Richter, and H. Lüth, Nano Lett. 7, 2166 (2007).

${ }^{17}$ P. D. C. King, T. D. Veal, C. F. McConville, J. Zuñiga-Pérez, V. Muñoz-Sanjosé, M. Hopkinson, E. D. L. Rienks, M. Fuglsang Jensen, and Ph. Hofmann, Phys. Rev. Lett. 104, 256803 (2010).

${ }^{18}$ G. Bridoux, M. Villafuerte, J. M. Ferreyra, J. Guimpel, G. Nieva, C. A. Figueroa, B. Straube, and S. P. Heluani, Appl. Phys. Lett. 112, 092101 (2018).

${ }^{19}$ H. Morkoç and Ü. Özgür, Zinc Oxide (Wiley-VHC, Weinheim, 2009).

${ }^{20}$ J. I. Pankove, Optical Processes in Semiconductors (Dover Publications Inc., New York, 1976).

${ }^{21}$ L. E. Halliburton, N. C. Giles, N. Y. Garces, M. Luo, C. Xu, L. Bai, and L. A. Boatner, Appl. Phys. Lett. 87, 172108 (2005).

${ }^{22}$ L. Liu, Z. Mei, A. Tang, A. Azarov, A. Kuznetsov, Q. -K. Xue, and X. Du, Phys. Rev. B 93, 235305 (2016).

${ }^{23}$ A. Janotti and C. G. Van de Walle, Appl. Phys. Lett. 87, 122102 (2005).

${ }^{24}$ A. Janotti and C. G. Van de Walle, Phys. Rev. B 76, 165202 (2007).

${ }^{25}$ K. Vanheusden, C. H. Seager, W. L. Warren, D. R. Tallant, and J. A. Voigt, Appl. Phys. Lett. 68, 403 (1996).

${ }^{26}$ L. L. Yang, Q. X. Zhao, M. Q. Israr, J. R. Sadaf, M. Willander, G. Pozina, and J. H. Yang, J. Appl. Phys. 108, 103513 (2010).

${ }^{27} \mathrm{~J}$. H. Davies, The Physics of Low-dimensional Semiconductors (Cambridge University Press, New York, 1998).

${ }^{\mathbf{2 8}}$ C. Lamberti, Characterization of Semiconductor Heterostructures and Nanostructures (Elsevier, Amsterdam, 2008).

${ }^{29}$ J. D. Dow and D. Redfield, Phys. Rev. B 1, 3358 (1970).

${ }^{30}$ C. Zapata, G. Nieva, J. M. Ferreyra, M. Villafuerte, L. Lanoël, and G. Bridoux, J. Phys. Condens. Matter 31, 345801 (2019).

${ }^{31}$ G. Bridoux, M. Villafuerte, J. M. Ferreyra, N. Bachi, C. A. Figueroa, and S. P. Heluani, Phys. Rev. B 92, 155202 (2015).

${ }^{32}$ M. W. Allen, C. H. Swartz, T. H. Myers, T. D. Veal, C. F. McConville, and S. M. Durbin, Phys. Rev. B 81, 075211 (2010).

${ }^{33}$ F. K. Shan, G. X. Liu, W. J. Lee, and B. C. Shin, J. Appl. Phys. 101, 053106 (2007).

${ }^{34}$ X. L. Wu, G. G. Siu, C. L. Fu, and H. C. Ong, Appl. Phys. Lett. 78, 2285 (2001).

${ }^{35}$ H. Kato, K. Miyamoto, M. Sano, and T. Yao, Appl. Phys. Lett. 84, 4562 (2004).

${ }^{36}$ M. D. Pashley, Phys. Rev. B 40, 10481 (1989).

37J. B. L. Martins, J. Andrés, E. Longo, and C. A. Taft, Int. J. Q. Chem. 57, 861 (1996).

${ }^{38}$ M. Kunat, St. Gil Girol, U. Burghaus, and Ch. Wöll, J. Phys. Chem. B 107, 14350 (2003)

${ }^{39}$ P. D. C. King, T. D. Veal, and C. F. McConville, Phys. Rev. B 77, 125305 (2008).

${ }^{40}$ X. Liu, X. Zhang, M. Bo, L. Li, H. Tian, Y. Nie, Y. Sun, S. Xu, Y. Wang, W. Zheng, and C. Q. Sun, Chem. Rev. 115, 6746 (2015).

${ }^{41}$ M. Schiek, K. Al-Shamery, M. Kunat, F. Traeger, and C. Wöll, Phys. Chem. Chem. Phys. 8, 1505 (2006)

${ }^{42}$ N. Bundaleski, A. G. Silva, U. Schroder, A. M. C. Moutinho, and O. M. N. D. Teodoro, J. Phys. Conf. Ser. 257, 012008 (2010).

${ }^{43}$ H. Lüth, Solid Surfaces, Interfaces and Thin Films (Springer, Heidelberg, 2010).

${ }^{44}$ A. Schleife, F. Fuchs, C. Rödl, J. Furthmüller, and F. Bechstedt, Appl. Phys. Lett. 94, 012104 (2009).

${ }^{45}$ E. Bauer, The Chemical Physics of Solid Surfaces and Heterogeneous Catalysis, edited by D. A. King and D. P. Woodruff (Elsevier, Amsterdam, 1983), Vol. 3. ${ }^{46}$ S. Ma, H. Liang, X. Wang, J. Zhou, L. Li, and C. Q. Sun, J. Phys. Chem. C 115, 20487 (2011). 